\title{
Quality of life outcomes after coronary artery bypass graft surgery: Relationship to neuropsychologic deficit
}

Everard W. Thornton, PhD, ${ }^{a}$ Christina Groom, PhD, ${ }^{a}$ Brian M. Fabri, MD, ${ }^{\text {b }}$ Mark A. Fox, MB, BS, ${ }^{\text {b }}$ Claire Hallas, PhD, ${ }^{c}$ and Mark Jackson, $\mathrm{PhD}^{\mathrm{b}}$

From the Department of Psychology, University of Liverpool, ${ }^{\text {a }}$ Cardiothoracic Centre, Liverpool NHS Trust, ${ }^{\mathrm{b}}$ Liverpool, and Harefield Hospital, ${ }^{\mathrm{c}}$ Middlesex, United Kingdom.

Support for this study was provided by the National Health Service Research and Development Fund, North West United Kingdom.

Received for publication Nov 12, 2004; revision received May 12, 2005; accepted for publication May 23, 2005.

Address for reprints: E. W. Thornton, PhD, Department of Psychology, University of Liverpool, Eleanor Rathbone Building, Bedford Street South, Liverpool L69 7ZA, United Kingdom (E-mail: ewt1 @ liverpool. ac.uk).

J Thorac Cardiovasc Surg 2005;130:1022-7

$0022-5223 / \$ 30.00$

Copyright () 2005 by The American Association for Thoracic Surgery

doi:10.1016/j.jtcvs.2005.05.020
Objectives: Neuropsychologic assessment after coronary artery bypass graft surgery indicates cognitive deficits, but data on their effect on health-related quality of life are relatively sparse. The present study assessed neuropsychologic deficits, selfreported health-related quality of life, and mood, together with proxy rating of patients' activities after bypass surgery. More specifically, the study examines the relationship between these varied outcome measures.

Methods: A prospective longitudinal assessment of 71 male patients was performed over a 6-month period: 1 week before surgery and postoperatively at 2 and 6 months. Within-patient change was assessed with the neuropsychologic test battery and procedures recommended by the Consensus Panel, the Short Form-36 to measure self-reported health-related quality of life, and the Hospital Anxiety and Depression Scale questionnaire to assess anxiety and depression. Proxy ratings were documented with the Functional Activities Questionnaire.

Results: Poor preoperative health-related quality of life was largely unrelated to medical variables. Cognitive deficit was found in $42 \%$ of patients at 2 months and $22 \%$ of patients at 6 months. Physical health-related quality of life improved, but benefit for emotional and social functioning was unconvincing, especially over the short term. Although cognitive deficits were largely unrelated to Short Form-36 health-related quality of life, and only partially related to anxiety and depression, they were associated with proxy ratings of patient functioning.

Conclusions: Physical health-related quality of life benefits were confirmed, but adverse cognitive change and only limited emotional benefit were evident after coronary artery bypass graft surgery. Perceived physical gains are unrelated to any cognitive deficit, but the latter correlated with the emotional status of the patient 6 months after surgery and are of concern to close relatives. These issues should be addressed in patient care.

$\mathrm{C}$ oronary artery bypass graft $(\mathrm{CABG})$ surgery with cardiopulmonary bypass (CPB) is performed to reduce cardiac mortality and to improve quality of life of the patient through relief of symptoms. ${ }^{1}$ However, there have been consistent reports of cognitive deficit in up to $80 \%$ of patients during the first 2 weeks after surgery, with a decline over subsequent weeks. Such deficits remain evident in $10 \%$ to $57 \%$ of patients 3 to 6 months after surgery. ${ }^{2,3}$ Such cognitive deficits seemingly contradict claims for improved self-reported health-related quality of life (HRQoL) outcomes after CABG surgery with CPB. ${ }^{4-6}$ With the Medical Outcomes Study Short Form-36 (SF-36), Lindsay and associates ${ }^{6}$ found improvement in physical functioning 3 years after surgery, with less convincing evidence of emotional benefit. However, studies have not universally reported positive HRQoL outcomes, ${ }^{7}$ and postoperative emotional changes, including depression, have been reported in $19 \%$ to $61 \%$ of patients. ${ }^{8}$ Less attention has been paid to anxiety, but 
estimates of postoperative anxiety range between $18 \%$ and $25 \% .{ }^{9}$ McKhann, ${ }^{10}$ Andrew, ${ }^{11}$ and their associates report no benefit from surgery in terms of mood, with anxiety actually increasing in the latter study. Self-report scales used in these studies may fail to capture change if compensatory social or other adjustments occur after surgery, although those close to the patient may retain an awareness of problem areas.

It is unclear to what extent the HRQoL and mood outcomes relate to cognitive deficits after surgery. Two previously cited studies reported no correlation between depression and cognitive change. ${ }^{10,11}$ The present study documents longitudinal self-reports and proxy reports of HRQoL outcomes and mood status after CABG surgery with $\mathrm{CPB}$ and examines whether such changes relate to cognitive deficits objectively assessed in neuropsychologic tests (NTs).

\section{Methods \\ Patients}

The patient sample consisted of 71 men, with a mean age 61 years $(\mathrm{SD}=8.5)$, undergoing elective CABG surgery. None had previously undergone cardiac surgery or had a psychiatric history or previous neurologic condition, including stroke, head injury, Parkinson's disease, or epilepsy. English was their native language, and they were not limited by sight or hearing problems with respect to test procedures; $53 \%$ had had a myocardial infarction, $62 \%$ had stable angina (grade 3), 85\% were married, and the average body mass index was 28 . Patients were excluded if their prognosis was poor (ejection fraction $<30 \%$; Coronary Artery Surgery Study left ventricular score $>16$ ) or they were older than 75 years. Women were excluded because of limited referrals and their wide demographic variance. Three-vessel disease was evident in $80 \%$ of the patients.

\section{Surgical Procedures}

All procedures were approved by the hospital ethics committee, and after provision of written information, consent forms were provided for both patients and their relative for signature at least 24 hours before data collection. Anesthesia was maintained by enflurane after fentanyl and diazepam induction. Surgical procedures were performed by the same cardiac surgeon and anesthetist. CPB was performed with an OPTIMA CML membrane oxygenator (COBE Cardiovascular, Inc, Arvada, Colo) using either moderate systemic hypothermia $\left(28^{\circ} \mathrm{C}-33^{\circ} \mathrm{C}\right)$ or normothermia $\left(37^{\circ} \mathrm{C}\right)$. During $\mathrm{CPB}$, blood pressure was maintained between 50 and 80 $\mathrm{mg} \mathrm{Hg}$, hematocrit between $18 \%$ and $30 \%$, and pump flow at 2.4 $\mathrm{L} \cdot \min \cdot \mathrm{m}^{2}$. Alpha-stat management of acid/base balance was used. Consecutive patients referred for surgery were randomly allocated to either normothermic or hypothermic surgical procedures with $\mathrm{N}=36$ in the former category. The surgeon was blinded to groups until onset of bypass.

\section{Test Procedures}

NTs, HRQoL, and anxiety and depression questionnaires were assessed preoperatively 1 week before surgery and subsequently repeated 2 and 6 months postoperatively according to the same schedule. Patients and their spouses were familiarized with
HRQoL and mood questionnaires in the hospital preoperatively, but these were given to patients to take home, complete, and return within 1 week. The same procedure was used after follow-up NT. The spouse or proxy provided feedback according to the same schedule and procedure. The Neuropsychologic Test Battery (NTB) was that recommended by the Consensus Panel for Neurobehavioral Outcomes after Cardiac Surgery. ${ }^{12}$ The tests, Rey Auditory Verbal Learning Test, Reitan Trail Making Test Form A, Reitan Trail Making Test Form B, Grooved Pegboard Test-Dominant Hand, and Grooved Pegboard Test-Nondominant Hand, provide 5 scores. A cognitive deficit on retest was defined on the 20:20 single case criterion recommended by the Consensus Panel, that is, an individual decline in performance from a preoperative baseline score of at least $20 \%$ on $20 \%$ of the test scores.

\section{Health-Related Quality of Life and Mood}

Two HRQoL questionnaires were used: a self-report and a proxy assessment. The self-report MOS SF- $36^{13}$ comprises 36 items that measure 8 multi-item dimensions: physical functioning, social functioning, role limitations because of physical functioning, role limitations because of emotional problems, mental health, energy and vitality, pain and general perception of health. Questions relating to pain were omitted. For each dimension scores are coded and averaged to provide a single score from 0 (worst possible health) to 100 (best possible health) for each dimension. The second scale, The Functional Activities Questionnaire (FAQ) ${ }^{14}$ is a proxy measure that was completed by the spouse or close friend or relative and assesses the ability of the patient to perform higher-level skills, termed "social functions." It is a 12-item scale, each scored on a scale of 1 to 3 with the high score representing a high level of dependence (poor health). Levels of anxiety and depression were assessed with the Hospital Anxiety and Depression Scale (HADS). ${ }^{15}$ The HADS is a self-report questionnaire that comprises 14 items split into 2 subscales, separately scored for anxiety and depression, and was originally designed for use in hospital outpatient departments. The scale has been extensively documented and validated. Each subscale has good internal consistency, and items relate to cognitions and anhedonia, with only a single somatic item. Each item is scored 0 to 3, and the threshold score for clinical case status is greater than 10 on each subscale, with scores of 8 to 10 providing borderline clinical status.

\section{Data Analysis}

Data are presented as an incidence analysis of the percentage of patients meeting the 20:20 criterion for NP test deficit. HRQoL data are initially presented as mean scores, but a similar criterion of an increase of $20 \%$ from preoperative levels was used to determine an individual incidence analysis of "significant" change. Statistical analyses were performed by the SPSS software package (SPSS Inc., Chicago, Ill).

\section{Results}

Examination of the data for different surgical temperature conditions indicated no difference between the 2 operative groups in outcomes, and statistical analyses confirmed neither large nor significant between-group effects. Data were therefore pooled across the 2 conditions for present analyses. 
TABLE 1. Percentage of patients reporting preoperative moderate to poor health (score $<50$ ) on each subscale of the Short Form-36 Quality of Life (health-related quality of life) questionnaire

\begin{tabular}{lcc}
\hline & n & Percentage \\
\hline Physical & 45 & 63.4 \\
Physical Role Limitations & 57 & 80.3 \\
Vitality & 45 & 63.4 \\
Social Functioning & 17 & 23.9 \\
General Mental Health & 13 & 17.4 \\
Emotional Role Limitations & 35 & 49.3 \\
General Health Perception & 38 & 53.5 \\
\hline
\end{tabular}

$n$, Number of patients reporting score $<50$.

\section{Neuropsychologic Test Battery}

The level of attrition of patients to the study over the follow-up was $\mathrm{N}=9$ at the 2-month follow-up and $\mathrm{N}=9$ at the 6-month follow-up. Examination of the data revealed no consistent relationship between patient attrition and performance on the preceding test occasion. The percentage of patients with NTB deficit defined by the 20:20 criterion was $42 \%$ at the 2-month follow-up and $22 \%$ at the 6-month follow-up. To better describe the changes in NTB outcomes over the 2 postoperative assessments, individuals who completed both assessments $(\mathrm{N}=52)$ were categorized according to 1 of 4 patterns: sustained impairment, no impairment, early impairment at the 2-month follow-up, and late impairment at the 6-month follow-up. The percentage of patients evidencing such cognitive deficits (20:20 criterion) for each of these patterns is $17.3 \%$ for sustained improvement, $53.9 \%$ for no improvement, $19.2 \%$ for early impairment at 2-month follow-up, and $9.9 \%$ for late impairment at 6-month follow-up.

\section{Medical Outcomes Study Short Form-36 Quality of Life Scale}

Each subscale dimension was dichotomized at the midpoint of the scale to represent good or poor HRQoL at each assessment. Scores less than 50 were interpreted as moderate to poor health. The percentage of patients self-reporting such status for each dimension at preoperative assessment is presented in Table 1. The figures vary with the greatest problems in physical role $(80.3 \%)$ and the least problems in general mental health (18.3\%). The mean scale scores for each dimension at preoperative and postoperative assessments are presented in Table 2 . These data were analyzed by analysis of variance with subsequent Bonferroni correction and level of statistical significance adjusted to $P$ less than .01 because of multiple comparisons. Although physical health, physical role limitation, and energy/vitality all improved from preoperative levels at both the 2- and 6-month follow-ups, there was no significant improvement at the 20-month follow-up for emotional role, social role, or general mental health. At the 6-month follow-up, there was some evidence of improvement for the latter 2 categories, with the greater significant improvement in social function.

The percentage of patients showing a substantial improvement at each postoperative assessment, defined by the criterion of $20 \%$ change relative to preoperative baseline, is presented in Table 3. These data indicate that a large percentage of patients improve in physical dimensions (physical role and vitality) with the minority of patients showing improvement in mental health and emotional role.

\section{Functional Activities Questionnaire}

The mean values for each assessment for the FAQ are provided in Table 2. Analysis of variance revealed no significant change across assessments.

\section{Anxiety and Depression}

Mean scores for both anxiety and depression, presented in Table 2, are below levels for clinical or borderline clinical status, with anxiety levels higher than those for depression. Analysis of variance revealed significant effects of time of assessment for both anxiety $(\mathrm{F}=6.2 ; P<.01)$ and depression $(\mathrm{F}=10.9 ; P<.001)$. Paired comparisons with Bonferroni correction confirmed significant reductions of both anxiety and depression scores between preoperative levels and those at the 6-month follow-up. Incidence analysis of the percentage of patients showing a $20 \%$ change from baseline is presented in Table 3 . The data show the majority of patients demonstrate an improvement in both anxiety and depression at the 2-month follow-up.

\section{Correlation Analyses}

Correlations (Spearman) were effected to examine the relationship between demographic and medical variables and preoperative performance on the NTB (deficit vs no deficit) and HRQoL variables. In addition, correlations were performed to examine postoperative relationships between the overall classification of the NTB and each of the mood and HRQoL assessments.

\section{Preoperative Status}

Outcome measures were correlated with 7 demographic/ medical variables: age, number of coronary arteries with occlusions, hypertension, diabetes (types I or II), shortness of breath (New York Heart Association class), angina grade (New York Heart Association class), and whether the patient was taking 6 or more different prescribed medications (polypharmacy). Age was the only variable significantly $(P<.01)$ associated with NTB performance, but age was not correlated with any of the HRQoL scales, except depression, at a marginal level of significance (rho $=0.21$, $P<.05)$. All of the self-reported dimensions of the SF-36 were correlated with angina. However, angina was not 
TABLE 2. Mean preoperative and postoperative scores for each questionnaire assessment: Self-report Short Form-36, anxiety and depression (Hospital Anxiety and Depression Scale), and proxy rating of functional activities (Functional Activities Questionnaire)

\begin{tabular}{lcccl}
\hline & Preoperative & $\begin{array}{c}\text { Two months } \\
\text { postoperative }\end{array}$ & $\begin{array}{c}\text { Six months } \\
\text { postoperative }\end{array}$ & $\begin{array}{c}\text { Significant } \\
\text { statistical comparison* }\end{array}$ \\
\hline Physical & 43.3 & 63.3 & 64.0 & $P<.01 ; .01$ \\
Physical Role Limitations & 19.6 & 28.2 & 51.8 & $\mathrm{NSt} ; P<.01$ \\
Vitality & 40.1 & 54.6 & 57.9 & $P<.01 ; .01$ \\
Social Functioning & 64.4 & 70.5 & 79.7 & $\mathrm{NS} ; P<.01$ \\
General Mental Health & 68.9 & 74.9 & 77.8 & $\mathrm{NS} ; \mathrm{NSt}$ \\
Emotional Role Limitations & 53.2 & 64.3 & 65.8 & $\mathrm{NS} ; \mathrm{NS}$ \\
General Health Perception & 46.9 & 65.9 & 58.9 & $P<.01 ; .01$ \\
Anxiety & 6.4 & 5.3 & 4.9 & $\mathrm{NS} ; P<.01$ \\
Depression & 4.7 & 3.4 & 2.6 & $\mathrm{NS} ; P<.01$ \\
FA0 & 2.45 & 1.78 & 2.12 & $\mathrm{NS} ; \mathrm{NS}$
\end{tabular}

FAQ, Functional Activities Questionnaire; NS, not significant. *Paired comparisons with Bonferroni correction and adjustment for significance at $P<.01$. Comparisons with preoperative assessment for both 2- and 6-month follow-ups. †NS $=$ Result would have been accepted at $P<.05$.

correlated with anxiety, depression, or the proxy FAQ questionnaire scores. The only other significant correlations were between the FAQ values and the number of coronary artery occlusions (rho $=0.3, P<.01$ ), and polypharmacy and several HRQoL subscale measures. These latter correlations were vitality (rho $=0.44, P<.01$ ), emotional role limitations (rho $=0.31, P<.01$ ), and general health perception (rho $=0.26, P<.05$ ). Depression (HADS) was also marginally positively associated with high levels of medication (rho $=0.25, P<.05$ ). In brief, NTB performance was correlated with age but not medical status. Inversely, self-reported HRQoL correlated with angina and overall medical severity as indexed by level of drug medication, but not with age.

\section{Postoperative Analyses of Neuropsychologic Test}

\section{Battery, Health-Related Quality of Life, and Mood}

Correlations were performed to relate cognitive deficit at either the 2-month follow-up or the 6-month follow-up test session with the likelihood of a significant change in the HRQoL dimension or change in anxiety or depression from preoperative levels at similar time points. Significant change for these variables was defined as a $20 \%$ change from the preoperative level of the specific subscale. Correlation of the 7 dimensions of the SF-36 with the NTB deficit at both the 2-month follow-up and 6-month follow-up revealed only 1 (of a possible 28) significant outcome (after Bonferroni correction and adjustment of $P<.01$ ): that between a change in physical role limitations and the 2-month follow-up NTB deficit (rho $=0.41)$. Correlations between the NTB deficit and change of mood were also not significant at the 2-month follow-up. However, at the 6-month follow-up there was significant association with both anxiety (rho $=0.28, P<.05)$ and depression (rho $=$ $0.23, P<.05$ ). Thus, a smaller long-term cognitive deficit
TABLE 3. Incidence analysis

\begin{tabular}{lcc}
\hline & $\begin{array}{c}\text { Two months } \\
\text { postoperative }\end{array}$ & $\begin{array}{c}\text { Six months } \\
\text { postoperative }\end{array}$ \\
\hline Physical & 62.9 & 65.5 \\
Physical Role Limitations & 27.9 & 45.4 \\
Vitality & 52.5 & 63.6 \\
Social Functioning & 45.2 & 47.3 \\
General Mental Health & 29.5 & 29.1 \\
Emotional Role Limitations & 38.3 & 32.7 \\
General Health & 54.8 & 54.5 \\
Anxiety & 59.7 & 59.3 \\
Depression & 59.7 & 66.7 \\
\hline
\end{tabular}

The percentage of patients who showed improvement from preoperative baseline at each postoperative follow-up. Data are provided for each dimension of the Short Form-36 quality of life questionnaire, anxiety and depression (Hospital Anxiety and Depression Scale).

seemed to be associated with a more positive long-term mood outcome. In contrast with self-rated SF-36 HRQoL, there were significant correlations between the proxy FAQ and cognitive deficits at both the 2- and 6-month follow-ups $(P<.01)$.

The variation in the outcome of these correlation analyses supports the notion that the scales are measuring different aspects of the postoperative life of the patient. Indeed, correlations between SF-36 dimensions and those of the HADS scales and FAQ show limited significant outcomes. Thus, of 56 possible correlations between SF-36 changes at the 2- and 6-month follow-ups and similar changes for anxiety and depression (HADS), only 2 attain the required level of significance $(P<.01)$. Although 4 of the 28 possible correlations between the FAQ change scores and those of the SF-36 suggest more commonality, the specific subscales of the SF-36 that do correlate are consistent. 
These are between FAQ and social functioning and emotional role limitations, with the suggestion that it is these aspects that most influence proxies in their ratings of limitation.

\section{Discussion}

The present study has documented neurocognitive and psychologic profiles of patients, comparing preoperative status with deficits and change at 2 and 6 months after CABG surgery. The data confirm a substantial number of patients have short-term (2-month) cognitive decline after CABG with $\mathrm{CPB}$, with $22 \%$ of patients still showing a significant deficit 6 months after surgery. These deficits are similar to those reported in extensive data from Duke University ${ }^{16}$ and in the midrange of those found in other studies, suggesting comparable patient group and procedures in the present study.

The SF-36 indicates poor preoperative HRQoL for patients undergoing $\mathrm{CABG}$ surgery relative to the general population. ${ }^{17}$ The low level of preoperative SF-36 subscales for physical function (physical, physical role, vitality) are comparable to those of patients who undergo cardiac surgery in the United States. ${ }^{18}$ Examination of SF-36 HRQoL data indicate that emotional role limitations and social functioning are less impaired preoperatively than physical dimensions (Table 1). The failure to show smaller improved postoperative emotional role limitations and general mental health relative to physical and vitality dimensions (Table 3 ) is consistent with other reports of outcomes for HRQoL using the SF-36, ${ }^{5,6}$ although 1 previous study noted no discrepancy. ${ }^{19}$

Changes in levels of anxiety and depression reflect the mental health and emotional role changes in SF-36 only in part. Despite low mean baseline scores, there is a gradual change from preoperative levels with improved postoperative mood status for the majority of patients even at the 2-month follow-up, although the change of mean scores for both anxiety and depression are not significant until the 6-month follow-up. The significant correlations between self-report scores of HADS anxiety and depression and SF-36 emotional role limitations are complementary and support the notion that these aspects provide problem areas for patients. However, although cognitive deficits are not related to emotional role limitations or mental health at either follow-up assessment, both anxiety and depression are associated with cognitive deficit at the later 6-month follow-up. These data on anxiety and depression at the 6-month follow-up are not consistent with those of other studies that indicate little relationship between changes in clinical negative mood states and cognitive decline after surgery. ${ }^{10}$

The proxy ratings of the FAQ fail to show favorable postoperative outcomes apparent for most SF-36 dimen- sions. This reflects an important psychologic outcome. Although the patient may realize the expected positive physical outcome from surgery, the unanticipated cognitive deficits and problems in emotional roles and general mental health give concern for the spouse or close relative/friend. More evidence on this issue is required because proxies have been reported more generally to assign a poorer HRQoL to patients than the patient themselves ${ }^{20}$ and to overestimate patient distress and emotional disturbance, whereas doctors may underestimate such severity. ${ }^{21}$ The significant correlation between proxy ratings and cognitive deficit suggests that these estimates reflect more than an overconcern for patients after major surgery.

There was little evidence from correlation analyses to suggest that patient's self-report of change of HRQoL as documented by the SF-36 was related to cognitive deficit objectively documented in NTB change. The absence of such contrasts with the significant correlation between NTB deficit and FAQ scores. One interpretation of this is that patients will acknowledge improvement of their HRQoL in self-report, especially their physical status, although their mood may be affected and they may be aware of cognitive changes after surgery. Indeed, this is our experience from patients referred for psychologic support in rehabilitation. As an example, a patient with decreased angina and dyspnea and improved stamina and activity after surgery may still be puzzled and disturbed by symptoms such as uncharacteristic tearfulness to "sad" events, such as a movie. These changes may also be noted with more concern by the spouse. Although this may not be of medical concern, and may not be revealed by generic HRQoL scales such as the SF-36, it may affect the core self-image of the patient. In the mind of the patient, and that of his or her spouse, the patient has "changed," and this may limit improvement of mood with implications for rehabilitation and the success of the operative procedure. Indeed, increased irascibility and irritability and rapid mood changes have been reported. ${ }^{22,23}$ The focus on NTB deficit as the primary outcome of CPB surgery, even if supplemented with generic HRQoL scales, fails to address such issues. Reduced cardiac morbidity and improved physical status may be salient for doctors, but cognitive and emotional role changes may leave the patient, and especially the proxy, less certain of the universality of a beneficial outcome.

\section{Conclusion}

The study shows that although improvement in emotional role and mental health is limited, the majority of patients show enhanced physical dimension scores for HRQoL after bypass surgery. Neuropsychologic deficits are not associated with such physical changes, but changes are more likely to be noticed by the patient's spouse or close relative, 
and they are often associated with patient anxiety and depression.

\section{References}

1. Solomon AJ, Gersh BJ. Management of chronic stable angina: medical therapy, transluminal coronary angioplasty, and coronary artery bypass graft surgery: lessons from randomized trials. Ann Intern Med. 1998; 128:216-23.

2. Keith JR, Puente AE, Malcolmson KL, Tartt S, Coleman AE, Marks HF. Assessing postoperative cognitive change after cardiopulmonary bypass surgery. Neuropsychology. 2002;16:411-21.

3. Selnes OA, Goldsborough MA, Borowicz LM, McKhann GM. Neurobehavioural sequelae of cardiopulmonary bypass. Lancet. 1999;353: 1601-6.

4. Duits AA, Boeke S, Taams MA, Passchier J, Erdman RAM. Prediction of quality of life after coronary artery bypass graft surgery: a review and evaluation of multiple recent studies. Psychosom Med. 1997;59: 257-68.

5. Sjoland H, Caidahl K, Wiklund I, Haglid M, Hartford M, Karlson BW, et al. Impact of coronary artery bypass grafting on various aspects of quality of life. Eur J Cardiothorac Surg. 1997;12:612-9.

6. Lindsay GM, Hanlon P, Smith LN, Wheatley DJ. Assessment of changes in general health status using the short form 36 questionnaire 1 year following coronary artery bypass grafting. Eur J Cardiothorac Surg. 2000;18:557-64.

7. Magni G, Unger HP, Valfre C, Polesel E, Cesari F, Rizzardo R, et al. Psychosocial outcome one year after heart surgery: a prospective study. Arch Intern Med. 1987;147:473-7.

8. Timberlake NK. Incidence and patterns of depression following coronary artery bypass graft surgery. J Psychosom Res. 1997;43:197-207.

9. Boudrez H, Denollet J, Amsel BJ, et al. Psychological status of patients before and after coronary artery bypass surgery. In: Walter PJ, editor. Quality of life after open heart surgery. Dordrecht: Kluwer Academic Publisher Group; 1992. p. 169-76.

10. McKhann GM, Borowicz LM, Goldsborough MA, Enger C, Selnes OA. Depression and cognitive decline after coronary artery bypass grafting. Lancet. 1997;349:1282-4.
11. Andrew MJ, Baker RA, Kneebone AC. Knight JL. Mood state as a predictor of neuropsychological deficits following cardiac surgery. J Psychosom Res. 2000;48:537-46.

12. Murkin JM, Newman SP, Stumpf DA, Blumenthal JA. Statement of consensus on neurobehavioral outcomes after cardiac surgery. Ann Thorac Surg. 1995;59:1289-95.

13. Ware JE, Sherbourne CD. The MOS SF-36 Short Form Health Survey (SF-36). Conceptual framework and item selection. Med Care. 1992; 30:473-81.

14. Pfeffer RI, Karosaki TT, Harrah CH, Chance JM, Filos S. Measurement of functional activities in older adults in the community. J Gerontol. 1982;37:323-9.

15. Zigmond A, Snaith RP. The Hospital Anxiety and Depression Scale. Acta Psychiatr Scand. 1983;67:361-70.

16. Newman MF, Kirchner JL, Phillips-Bute B, Gaver V, Grocott H, Jones $\mathrm{RH}$, et al. Longitudinal assessment of neurocognitive function after coronary-artery bypass surgery. $N$ Engl J Med. 2001;344:395-402.

17. Jenkinson C, Coulter A, Wright L. Short form 36 (SF-36) health survey questionnaire: normative data for adults of working age. $\mathrm{Br}$ Med J. 1993;306:1437-44.

18. Yun KL, Sintek CF, Fletcher AD, Pfeffer TA, Kochamba GS, Mahrer PR, et al. Time related quality of life after elective cardiac operation. Ann Thorac Surg. 1999;68:1314-20.

19. Immer FF, Berdat PA, Immer-Bansi AS, Eckstein FS, Muller S, Saner $\mathrm{H}$, et al. Benefit to quality of life after off-pump versus on-pump coronary bypass surgery. Ann Thorac Surg. 2003;76:27-31.

20. Sneeuw KC, Aaronson NK, Sprangers MA, Detmar SB, Wever LD, Schnoragel JH. Comparison of patient proxy EORTC QLQ-C30 ratings in assessing the quality of life of cancer patients. J Clin Epidemiol. 1998;51:17-31.

21. Sprangers MAG, Aaranson NK. The role of health care providers and significant others in evaluating the quality of life of patients with chronic disease. J Clin Epidemiol. 1992;45:743-60.

22. Vingerhoets G, De Soete G, Jannes C. Subjective complaints versus neuropsychological test performance after cardiopulmonary bypass. J Psychosom Res. 1995;39:843-53.

23. Vingerhoets G. Cognitive, emotional and psychosomatic complaints and their relation to emotional status and personality following cardiac surgery. Br J Health Psychol. 1998;3:159-69.

\section{Bound volumes available to subscribers}

Bound volumes of The Journal of Thoracic and Cardiovascular Surgery are available to subscribers (only) for the 2005 issues from the Publisher, at a cost of $\$ 134.00$ for domestic, $\$ 165.85$ for Canadian, and $\$ 155.00$ for international subscribers for Vol 129 (January-June) and Vol 130 (July-December). Shipping charges are included. Each bound volume contains a subject and author index.

The binding is durable buckram with the Journal name, volume number, and year stamped in gold on the spine. Payment must accompany all orders. Contact Elsevier Inc., Subscription Customer Service, 6277 Sea Harbor Dr, Orlando, FL 32887, USA; phone 800-654-2452 or 407-345-4000.

Subscriptions must be in force to qualify. Bound volumes are not available in place of a regular Journal subscription. 